\title{
Perspectiva histórica de la enfermedad mamaria y su tratamiento
}

\author{
José Joaquín Caicedo Mallarino MD*
}

Las enfermedades mamarias y su tratamiento datan desde hace muchos años. El registro médico más antiguo se encuentra en uno de los Papiros Egipcios adquiridos por Edwin Smith. Se cree pertenece al año 1600 a.JC, y en el caso número 45 , relata un caso de CANCER DE SENO, para lo cual, decía, no hay tratamiento.

Se sabe que Hipócrates, quien contribuyó en gran forma con los avances de la medicina, describió 1 caso, pero no recomendaba tratamiento. A Leonidas, médico griego, se le atribuye el primer tratamiento quirúrgico descrito para un Cáncer de seno, con múltiples incisiones y cauterio hasta erradicar el tumor. Aurelius, Cornelius, Celsus intentan una clasificación clínica. Galeno, el médico más importante después de Hipócrates, describe el cáncer de seno como "una dolencia con venas distendidas que recordaban las patas de un cangrejo". Decía haber curado los casos tempranos, y recomendaba dejar sangrar a la paciente para eliminar "la bilis negra". Predominaba en la época la teoría de los Humores de Hipócrates.

La patrona de las enfermedades mamarias, patrona de las pacientes mastectomizadas es SANTA AGUEDA, a quien se le amputaron sus 2 senos, con unas tenazas de hierro al rojo vivo, por haber rechazado los amores del cónsul de su época, a mediados del siglo tercero.

En las culturas de la Edad Media, la cirugía era la combinación del cuchillo con el cauterio y era practicada por Barberos y hombres con poca educación; esta situación continuó hasta el Renacimiento. Con el descubrimiento de América, y la aparición de la prensa escrita comenzó a mejorar la enseñanza médica en varias universidades. Se practican autopsias, y surgen los grandes anatomistas como Vesalio, que revolucionan los conceptos de Cirugía. Se comienza a emplear las ligaduras en reemplazo del cauterio. En este campo se destacó Ambrose Pare, como uno de los más grandes cirujanos del Renacimiento. El doctor Wilhelm Fabry desarrolla un instrumento que facilita la amputación de seno. Sin embargo era muy alta la Mortalidad e infección de los pacientes. Hacia fines del siglo XVII aparecen las publicaciones médicas que difunden la información. En el siglo octavo, se introducen nuevos conceptos en cuanto a Fisiología y Patología y se comienzan a diferenciar los barberos de los cirujanos. John Hunter se le conoce como el padre de la cirugía experimental. Henri le Dran habla de la diseminación linfática del cáncer y acaba con la teoría de los

* Cirujano Oncólogo. Grupo de Cirugía General y Seno. Instituto Nacional de Cancerología. humores. Su alumno Jean Louis Petit, se puede considerar como el pionero de la mastectomía radical.

En el siglo diez y nueve se revoluciona la cirugía con la aparición de la anestesia y la antisepsia. Se comienza a disminuir la mortalidad y morbilidad. Sir James Paget describe en 1874 la enfermedad que lleva su nombre. Charles Moore recalca la importancia de la radicalidad, y decía que durante la cirugía el tumor nunca debía ser visto.

El doctor Samuel Gross y su hijo hicieron importantes aportes a la cirugía del seno. Hablaban de la cirugía en bloque incluyendo la fascia de pectoral y el contenido axilar. En Estados Unidos hospitales de Philadelphia y el John Hopkins en Baltimore se abren paso. Fue en este hospital en donde el Dr. William Stewart Halsted, realizó sus principales investigaciones. El completó sus estudios en Yale en 1874. Viajó a Europa en donde trabajó con el doctor Billroth, de quien aprendió mucho acerca del manejo del cáncer mamario. Regresó a Nueva York y en 1882 en el hospital Roosevelt realiza su primera cirugía RADICAL, que desde entonces se conoce con su nombre. En 1894 describe sus primeros 50 casos; no reporta mortalidad operatoria, y la sobrevida a 3 años fue del $45 \%$. Su cirugía incluía el concepto en BLOQUE, con los músculos pectorales. El doctor Willy Meyer en 1894, tan sólo 10 días después del doctor Halsted, publicó una técnica de mastectomía casi idéntica, pero comenzaba la disección por la axila.

En la primera década del siglo veinte, varios cirujanos comienzan a describir sus experiencias con la Mastectomía Radical. El doctor J. Collins Warren, describe el tratamiento quirúrgico para las recaídas locales, prolongando la sobrevida de algunas pacientes. William Handley contribuye al entendimiento de la diseminación metastásica, a partir de los ganglios linfáticos, concepto básico de la cirugía radical de Halsted. Se comienzan a hacer algunas modificaciones a la incisión original. El Dr. F.T Stewart diseña la incisión transversa, la cual utilizamos hoy en día. Con los estudios del doctor Handley dijo sobre la importancia de los ganglios de la mamaria interna y mediastino, algunos cirujanos a mediados de siglo, comienzan a practicar las llamadas mastectomías supraradicales, que incluían fosa supraclavicular, mamaria interna y mediastino (Urban, Margottini), pero pronto la alta mortalidad y morbilidad, les hizo abandonar la técnica. Paralelamente se desarrolla la radioterapia. Geoffray Keynes comienza a tratar pacientes con estados avanzados, con algún éxito. Es uno de los primeros en recomendar que se debe combinar con la cirugía en 
algunos casos. El doctor Gushman Haagensen de Nueva York, dedicó la vida al estudio de la patología mamaria. Fue siempre partidario de la cirugía radical. Describió claramente los criterios de inoperabilidad que todavía empleamos. Fue uno de los primeros en proponer la importancia del autoexamen de seno. En 1948, el doctor D. H. Patey, cirujano inglés, consideró que no era necesario resecar el músculo pectoral mayor, y llamó a su técnica Mastectomía Radical Modificada. En su publicación también describe 10 casos de cirugía conservadora, pero por la alta recidiva local abandona la técnica. El doctor Auchincloss en Nueva York y el doctor Madden, practican la técnica conservando ambos pectorales, técnica que utilizamos hoy en día.

El uso de la Mastectomía radical declina hasta casi desaparecer el día de hoy. La conferencia de consenso de 1980 concluye que la Mastectomía Radical Modificada es una técnica adecuada para los estados tempranos. Comienzan interrogantes sobre cirugía aun más conservadora, y Crile y col practican varias, pero con alto índice de recidiva local.

Se demuestra la importancia de agregar radioterapia al tratamiento conservativo. El National Surgical Adyuvant Breast and Bowel Project (NSABP) comandado por el Dr. Bernard Fisher, con su estudio B-06 confirma como el tratamiento conservador seguido de radioterapia, en casos bien seleccionados, da los mismos resultados en cuanto a sobrevida total, que la cirugía radical. Paralelamente viene trabajando el doctor Humberto Veronesi y su equipo en Milán, este último manejando el concepto de Cuadrantectomía. Otros autores en diferentes latitudes confirman lo mismo. Las teorías Halstedianas perduran por casi 75 años. El Dr. Bernard Fisher con lo que el llamó teoría alternativa, pero que creo se debe llamar Teoría de Fisher, demostró que el cáncer mamario es una enfermedad sistémica desde el principio, que los ganglios no son barreras efectivas sino que su compromiso implica la capacidad del tumor para dar metástasis; que existe una relación huésped-tumor muy importante. Sus estudios comienzan a demostrar y desarrollar campos como la Quimioterapia. Hay claros avances en cuanto a Radioterapia,
Hormonoterapia, Radiología (mamografia), Inmunoterapia, Cirugía plástica, Rehabilitación, Psiquiatría etc., que hacen que el manejo del Cáncer del seno es hoy en día MULTIDISCIPLINARIO.

El doctor George Thomas Beatson, en 1896 publicó una serie de 3 casos de pacientes con cáncer de seno que habían respondido a la OOFORECTOMIA. Se comienza también a emplear la radioterapia para tal fin. La era moderna de la cirugía endocrina retoma interés en 1954, con la castración femenina como parte del tratamiento en la mujer premenopáusica. Se practican adrenalectomías, y hasta hipofisectomías durante muchos años. En 1973 se descubren los receptores para estrógenos y progesterona (1975), permitiendo hoy en día la manipulación hormonal de muchos de los tumores y de la enfermedad metastásica. El Dr. Paul Ehrlich (1854-1915) se le conoce como 'el padre de la quimioterapia'. Durante la II Guerra Mundial se desarrolla la mostaza nitrogenada, y luego varios derivados: Múltiples estudios demuestran los resultados de la combinación de varias drogas, en el tratamiento del cáncer mamario, entre los que se destacan los del NSABP en EEUU, y en Milán los de Bonadonna al igual que otros países europeos, y que continúan en curso. Lo que hasta ahora ha demostrado la quimioterapia es que mejora la sobrevida libre de enfermedad, aunque la sobrevida total se ha alterado poco.

La cirugía cada vez tiende a ser menos radical. Sinembargo, en nuestro medio la Mastectomía Radical Modificada sigue siendo la que predomina. Hoy en día las técnicas cada vez mejores de reconstrucción nos abren nuevas perspectivas en el manejo quirúrgico del cáncer de seno, especialmente en cuanto a el concepto de CALIDAD DE VIDA.

E1 Dr. Fisher comenta. "Las estrategias terapéuticas para el manejo del cáncer de seno, han revolucionado en el tiempo, paso a paso, y son el resultado de información biológica, que ha llevado a un mejor entendimiento de la enfermedad. Es lógico anticiparse a que esto continuara, y los logros futuros se basarán en hipótesis biológicas nuevas. El cáncer de seno fue, es y será siempre relacionado a la Ciencia y no al populismo". 